\title{
EFFICIENT REACTIVE POWER COMPENSATION ALGORITHM FOR DISTRIBUTION NETWORK
}

\author{
J. Jerome \\ Electrical and Electronics Engineering Department, \\ PSG College of Technology, Coimbatore, Tamilnadu, India \\ E-mail: jjovitha@yahoo.com
}

Received 29 May 2003, Accepted 15 September 2003

\begin{abstract}
The use of automation and energy efficient equipment with electronic control would greatly improve industrial production. These new devices are more sensitive to supply voltage deviation and the characteristics of the power system that was previously ignored are now very important. Hence the benefits of distribution automation have been widely acknowledged in recent years. This paper proposes an efficient load flow solution technique extended to find optimum location for reactive power compensation and network reconfiguration for planning and day-to-day operation of distribution networks. This is required as a part of the distribution automation system (DAS) for taking various control and operation decisions. The method exploits the radial nature of the network and uses forward and backward propagation technique to calculate branch currents and node voltages. The proposed method has been tested to analyze several practical distribution networks of various voltage levels and also having high $\mathrm{R} / \mathrm{X}$ ratio.
\end{abstract}

Keywords : Distribution automation system, Reactive Power Compensation, Network Reconfiguration,Power Quality, Deregulated Power

\section{INTRODUCTION}

The power business is moving into new territory with market deregulation. Deregulation of the power industry has made power quality a distinguishing feature of distribution service. DAS is the key to address all these challenges to improve the operation of the distribution system and the quality of supply. Power system operators ensure the quality and reliability of supply to the customers by maintaining the load bus voltages within their permissible limit. Load flow is an important tool for the analysis of any power system. It is used in the operational as well as planning stages. The application programs like the network configuration and reactive power compensation require a robust and efficient load flow solution method. DAS is essential for efficient operation of the distribution networks. In view of the increasing use of SCADA, and distribution automation and control (DAC), distribution system reconfiguration becomes a more viable alternative for loss reduction. The networks are reconfigured to reduce system real power 
loss, optimal linking of new loads, relieve overloads, and service restoration. Reactive power compensation improves the voltage profile at the user terminal and improves the quality of supply. It also reduces the losses and provides economic benefit to the supplier.

Many researches have addressed distribution network reconfiguration and reactive power compensation proposing different approaches. The optimum operating condition can be considered to be obtained when the network presents (i) minimum losses, (ii) minimum voltage deviations at the consumer feeding points, and (iii) maximum reliability. A branch exchange type, heuristic algorithm has been suggested. A filtering mechanism has also been suggested to reduce the number of candidate switching options ${ }^{1}$. A power flow based heuristic algorithm for determining the minimum loss configuration of radial distribution networks has been reported ${ }^{2}$. Capacitor placement problem on radial distribution systems is formulated and a solution algorithm is proposed. The problem is formulated as a mixed integer-programming problem ${ }^{3}$. The problem is formulated and solved using a quadratic integer programming based approach to determine the number, locations and sizing of capacitors to be placed in the distribution systems. The proposed method does not take into account the voltage constraint ${ }^{4}$. Distribution networks are radial and $\mathrm{r} / \mathrm{x}$ ratio of lines is high. Conventional load flow methods based on Gauss-siedel and Newton-Rapson techniques are inefficient in solving distribution networks. An efficient and robust three-phase power flow algorithm for application to radial distribution networks was presented ${ }^{5}$. It uses the forward and backward propagation technique to calculate branch currents and node voltages. This method has been extended to network reconfiguration and reactive power compensation for application to the distribution networks.

\section{METHODOLOGY}

Distribution systems are usually unbalanced due to unbalanced loading of the different phases. This requires detail modeling of the special feature such as multiphase, grounded or ungrounded, unbalanced distribution loads and so on. The distribution feeders consist of three-phase overhead lines or underground cable sections. These have double-phase or single-phase line sections towards the end of the feeder. In the three-phase power flow algorithm the line branch has been model such that each node or line section in the network is numbered by a single index, regardless of the number of phases of this node or line or line section. The three-phase balanced/ unbalanced loads can be represented as either constant power, or constant current, or constant impedance type or a combination of these based on the proportion of the type of consumer loads. There is no limitation on the type of load to be modeled in the proposed algorithm. The load flow solution is found using methods of network flow (paths) along the tree in forward direction and backward direction. Figure 1 shows the computational blocks in the algorithm.

\section{COMPUTATIONAL ITERATIVE SCHEME}

For a given network, first the nodes are reordered in such a way as to generate proper source node (Parent node) and load node (child node) paths. This is explained in block A. A table of parent nodes and child nodes is created. A source node (parent node) can be connected to 


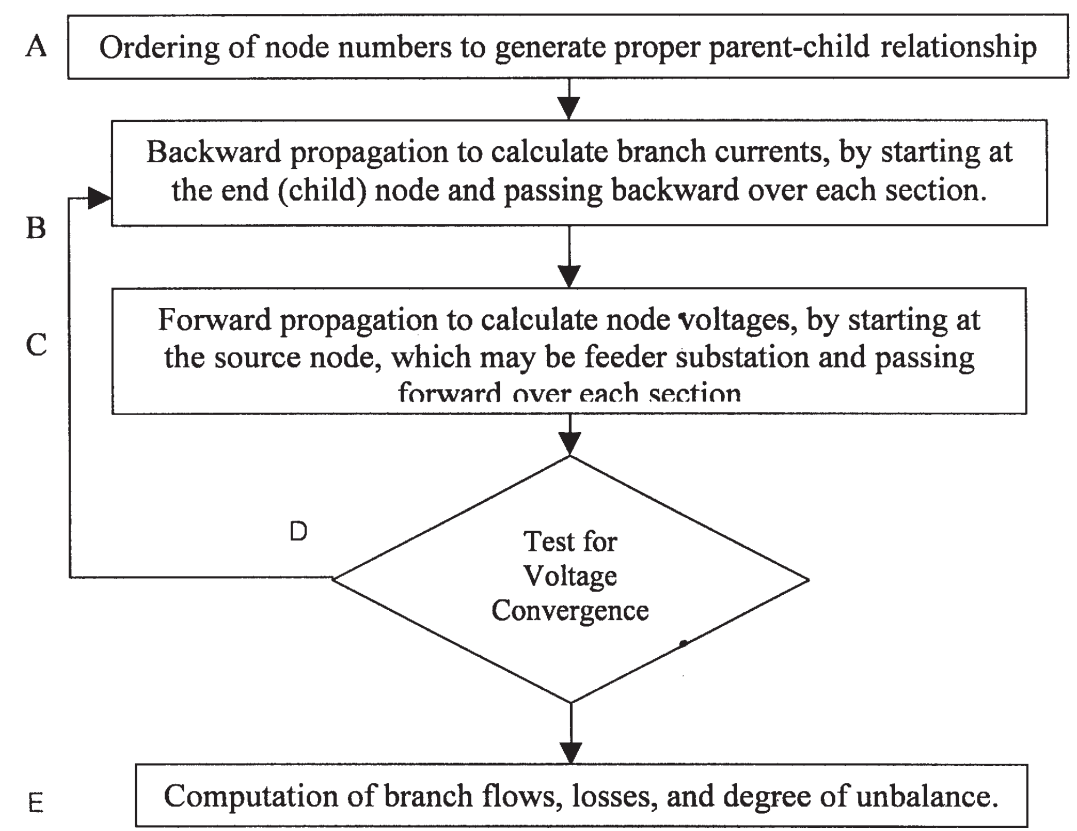

Figure 1 : Basic computational blocks in the proposed algorithm

more than a single load node (child node). A parent node can have more child nodes (children). But a load node (child node) has only one source node (parent node). This is due to radial nature of the distribution network. Each section of a radial distribution feeder is connected radially so that it has one predecessor (parent node) and possibly multiple descendants (Children nodes).

A backward walk is achieved by starting at the end nodes (Children nodes) and passing 'backward' over each section. During the backward propagation branch currents are calculated as explained in computational block B. The block $\mathrm{C}$ of the basic steps in the proposed algorithm, a forward walk is achieved by starting at the source that may be either the substation or the feeder and passing "forward" over each section. The node voltages are calculated. voltage convergence is executed in the block D. Computation of branch flows, losses, and degree of unbalance is done in computational block E. Node renumbering is the process of generating new node numbers for feeder nodes, which are initially numbered, arbitrarily. This is very useful particularly when the network is reconfigured using various switching options to meet the demand during different configuration.

Ordering of node numbers to generate proper parent-child relationship is known as the node renumbering process. The branch sequence of forward and backward paths is prepared. The optimally-ordered feeder nodes with new numbers starting from the source (parent) node and propagating in the forward path to load (child) node is obtained. A table is arranged to give the forward path from the source (parent) node to the load (child) node and backward path from the load (child) node to the source (parent) node. From the table the backward path and the forward path are created and stored in memory for further calculation ${ }^{5}$. 
Initially, all the node voltage magnitudes are set to 1.0 p.u. and voltage angles are set to 0.0 , $-120,120$ degrees for phase A, phase B and phase C, respectively. And also all the branch currents (complex) are initialized to $(0.0,0.0)$ p.u. The source node phase voltage magnitudes are assumed to be known, also its angles are $\delta_{\mathrm{a}}=0, \delta_{\mathrm{b}}=-120^{\circ}, \delta_{\mathrm{c}}=120^{\circ}$ (taken as reference). During the backward propagation the load current is calculated depending on the load type. The half line charging shunt currents of all the branches at the node are added to the load current. Once the child node current is calculated, the parent branch current is updated using the Equation (1) and considering that the loads are unbalanced constant power,

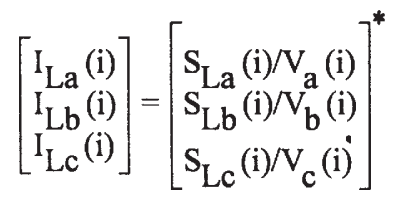

Where $\mathrm{I}_{\mathrm{La}}(\mathrm{i}), \mathrm{I}_{\mathrm{Lb}}(\mathrm{i}), \mathrm{I}_{\mathrm{Lc}}(\mathrm{i})=$ Load current at $\mathrm{i}^{\text {th }}$ node for constant power loads; $\mathrm{S}_{\mathrm{La}}(\mathrm{i}), \mathrm{S}_{\mathrm{Lb}}(\mathrm{i}), \mathrm{S}_{\mathrm{Lc}}(\mathrm{i})$ $=$ Complex power of constant power load at $\mathrm{i}^{\text {th }}$ node.

The branch current of the line sections is calculated using the equation (2).

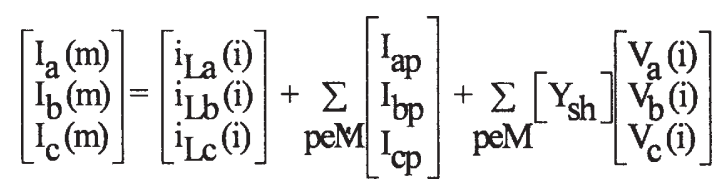

The purpose of forward propagation is to calculate the voltage and load at each node starting from the source node of the feeder. The feeder substation source voltage is set to its actual value. During forward propagation, the current in each branch is held constant to the value obtained in backward walk. The node voltages are calculated using the equation (3), starting from feeder source and proceeding along the forward

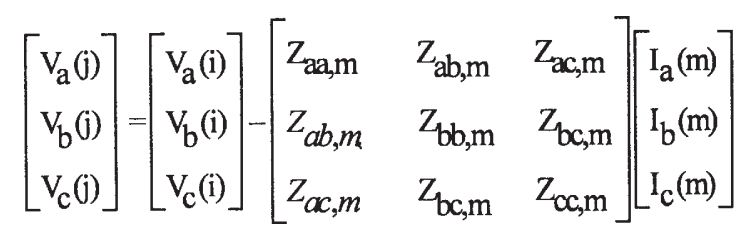

path. The convergence criteria are that the complex voltage real and imaginary parts at each node in all the three-phases are compared with their previous iteration values. Therefore the voltage mismatch for $\mathrm{j}^{\text {th }}$ node during $\mathrm{k}^{\text {th }}$ iteration is given by following Equation 4 .

$$
\begin{aligned}
& \triangle \mathrm{V}^{\mathrm{k}}(\mathrm{j})=\mathrm{V}^{\mathrm{k}}(\mathrm{j})-\mathrm{V}^{\mathrm{k}-1}(\mathrm{j}) \text { for } \mathrm{a}, \mathrm{b} \text {, and } \mathrm{c} \text { phases } \\
& \text { Real }|(\triangle \mathrm{V}(\mathrm{j}))|<\text { eps, } \mathrm{j} \varepsilon \text { all the nodes } \\
& \text { Imag }|(\triangle \mathrm{V}(\mathrm{j}))|<\text { eps, } \mathrm{j} \varepsilon \text { all the nodes }
\end{aligned}
$$

If both the conditions in Equations 5 and 6 are satisfied, the iterative process is stopped. Once the load flow solution is converged all the branch currents and voltage at each node are known. The real and reactive power flows and loss can be calculated. 


\section{NETWORK RECONFIGURATION}

The proposed load flow technique is extended to network reconfiguration, as it is efficient and robust for analyzing larger distribution system with higher number of nodes. First we can define the switching option with the available switches and total system losses can be calculated for each switching option. These loss values are arranged in merit order to obtain the optimum configuration. One of the important functions of the distribution automation is configuration management. In a normal state, configuration management deals with the change of feeder configurations through remotely controlled switches. In a fully automated system, all switches would be remotely controllable and all feeder loads can be monitored from the dispatching center. Thus the dispatcher would be able to transfer loads from one feeder to another while minimizing interruptions to customers. When a significant change of load is detected, a lossminimization algorithm can help the dispatcher generate a minimum-loss configuration. The distribution networks control was traditionally restricted to avoid overloading and voltage limit violations, under normal operating conditions. In recent years, the development in computer software and hardware has made the optimization of the network operation feasible.

\section{REACTIVE POWER COMPENSATION}

The application of the proposed load flow solution method has been tested for reactive power compensation and network reconfiguration in distribution system. The candidate location for reactive power compensation can be defined as the location where the feeder loss is found to be the lowest. This location is considered as the feeding source. The load flow is performed for a single iteration considering each node as possible feeding node and the losses are calculated. Then these losses are arranged in ascending order. The node, which is at the top of the merit order, is the optimum feeding node and it is the best location for reactive power compensation. Due to some geographical or other reason, if the first node in order is not suitable, the next node in the list is selected.

\section{SYSTEM STUDIES AND RESULTS}

A computer program has been developed based on the proposed algorithm and tested on a few distribution networks. Two typical systems, a 19-node practical system and a 38-node practical distribution network with multi-feeders are presented.

\subsection{9-node practical system}

The 19-node feeder is analyzed to find the merit order of the feeding nodes for load demand factor of 0.6. The loads were assumed balanced and load flow is performed for single iteration. The merit order for the given loading condition is presented in Table 1. The loss values shown are for single iteration of the load flow. The total loss at node 10 is $41.466 \mathrm{~kW}$ and the loss percentage is 3.29 . The node 10 is the best location for reactive power compensation. The node 3 is the worst location for reactive power compensation. The total loss at node 3 is $322.584 \mathrm{~kW}$ and the loss percentage is 20.91. Figure 2 presents the merit order with every node as feeding 


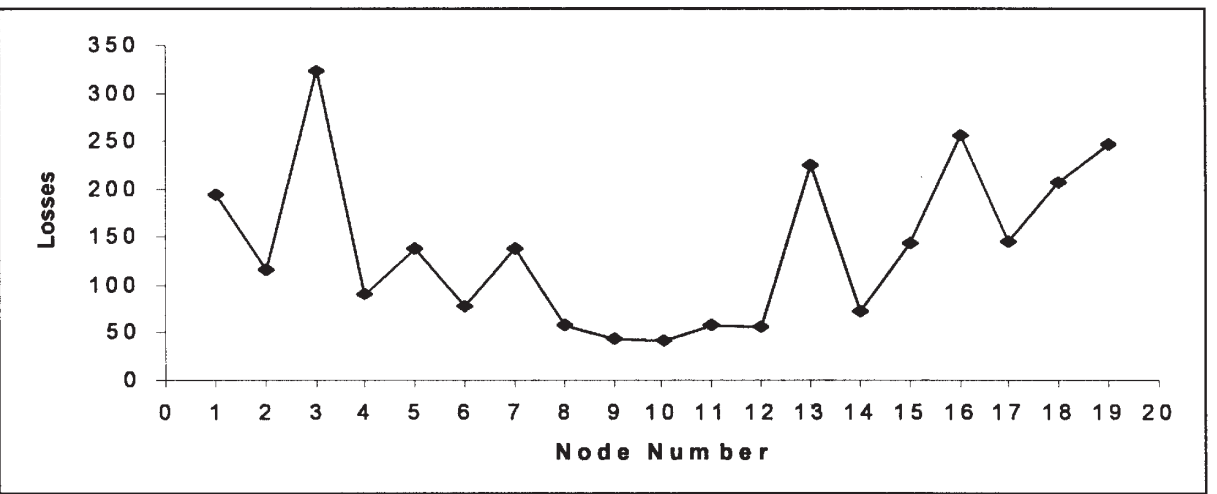

Figure 2: Merit order of the feeding points of 19-node system

Table 1 : Merit order of the feeding point for the 19-node system

\begin{tabular}{|c|c|c|c|c|c|c|c|}
\hline $\begin{array}{c}\text { Merit } \\
\text { order }\end{array}$ & $\begin{array}{c}\text { Node } \\
\text { No. }\end{array}$ & $\begin{array}{c}\text { Total Losses } \\
(\mathrm{kW})\end{array}$ & $\%$ Loss & $\begin{array}{c}\text { Merit } \\
\text { order }\end{array}$ & $\begin{array}{c}\text { Node } \\
\text { No. }\end{array}$ & $\begin{array}{c}\text { Total Losses } \\
(\mathrm{kW})\end{array}$ & $\%$ Loss \\
\hline 1 & 10 & 41.466 & 3.29 & 11 & 5 & 138.074 & 10.17 \\
\hline 2 & 9 & 43.128 & 3.41 & 12 & 15 & 142.558 & 10.46 \\
\hline 3 & 12 & 55.803 & 4.37 & 13 & 17 & 145.060 & 10.63 \\
\hline 4 & 11 & 57.274 & 4.48 & 14 & 1 & 193.897 & 13.72 \\
\hline 5 & 8 & 58.806 & 4.60 & 15 & 18 & 207.327 & 14.53 \\
\hline 6 & 14 & 72.665 & 5.62 & 16 & 13 & 225.178 & 15.58 \\
\hline 7 & 6 & 78.434 & 6.04 & 17 & 19 & 247.471 & 16.87 \\
\hline 8 & 4 & 90.802 & 6.93 & 18 & 16 & 255.966 & 17.34 \\
\hline 9 & 2 & 115.951 & 8.68 & 19 & 3 & 322.584 & 20.91 \\
\hline 10 & 7 & 136.964 & 10.09 & & & & \\
\hline
\end{tabular}

point. As a single iteration is performed, the CPU time taken for a bigger system will also be in the range of millisecond. Reactive power compensation is provided at the best and worst nodes. The percentage loss without compensation is $13.715 \%$. The candidate location for reactive power compensation is the node number 10 . The node 10 is the location where the feeder loss is found to be the lowest. This location is considered as the feeding source. About $250 \mathrm{kvar}$ compensation is placed at node 10 . The load flow is performed for a single iteration. The forward flows and losses are given in Table 2. The percentage loss with compensation at node 10 is $11.555 \%$. Reactive power compensation of about $250 \mathrm{kvar}$ is placed at the worst node 3. The load flow is performed for a single iteration. The forward flows and losses are given in Table 3. The percentage loss with compensation at node 3 is $13.365 \%$. 
Table 2 : Forward flows and losses with reactive power compensation at the candidate location (node 10)

\begin{tabular}{|c|c|c|c|c|c|}
\hline \multirow{2}{*}{ Node From } & Node & \multicolumn{2}{|c|}{ Forward Flows } & \multicolumn{2}{c|}{ Losses } \\
\cline { 3 - 6 } & To & $\mathrm{kW}$ & $\mathrm{kvar}$ & $\mathrm{kW}$ & $\mathrm{kvar}$ \\
\hline 1 & 2 & 1379.234 & 408.989 & 53.395 & 22.972 \\
\hline 2 & 3 & 87.057 & 42.032 & 3.657 & 2.187 \\
\hline 2 & 4 & 1152.380 & 302.138 & 19.871 & 8.549 \\
\hline 4 & 5 & 54.078 & 26.176 & 0.079 & 0.022 \\
\hline 4 & 6 & 1024.429 & 241.259 & 10.720 & 4.612 \\
\hline 6 & 7 & 86.677 & 41.924 & 0.276 & 0.078 \\
\hline 6 & 8 & 893.016 & 178.248 & 20.509 & 8.824 \\
\hline 8 & 9 & 818.506 & 143.270 & 21.511 & 9.255 \\
\hline 9 & 10 & 661.995 & 68.632 & 24.314 & 10.461 \\
\hline 10 & 11 & 285.255 & 137.915 & 1.786 & 0.768 \\
\hline 10 & 12 & 318.407 & 153.781 & 2.224 & 0.957 \\
\hline 11 & 14 & 142.740 & 69.055 & 0.302 & 0.130 \\
\hline 11 & 13 & 54.329 & 26.247 & 0.329 & 0.094 \\
\hline 12 & 16 & 87.423 & 42.136 & 1.023 & 0.291 \\
\hline 12 & 15 & 142.360 & 68.842 & 1.503 & 0.647 \\
\hline 14 & 17 & 54.152 & 26.219 & 0.153 & 0.066 \\
\hline 14 & 18 & 54.264 & 26.228 & 0.264 & 0.075 \\
\hline 15 & 19 & 86.858 & 42.042 & 0.457 & 0.197 \\
\hline
\end{tabular}

Table 3 : Forward flows and losses with reactive power compensation at the worst location (node 3)

\begin{tabular}{|c|c|c|c|c|c|}
\hline \multirow{2}{*}{$\begin{array}{c}\text { Node } \\
\text { From }\end{array}$} & Node & \multicolumn{2}{|c|}{ Forward Flows } & \multicolumn{2}{|c|}{ Losses } \\
\cline { 3 - 6 } & $\mathrm{kW}$ & $\mathrm{kvar}$ & $\mathrm{kW}$ & $\mathrm{kvar}$ \\
\hline 1 & 2 & 1408.050 & 420.946 & 55.723 & 23.974 \\
\hline 2 & 3 & 89.991 & 207.136 & 3.591 & 1.020 \\
\hline 2 & 4 & 1175.935 & 562.261 & 23.830 & 10.252 \\
\hline 4 & 5 & 54.080 & 26.176 & 0.079 & 0.023 \\
\hline 4 & 6 & 1044.028 & 499.681 & 13.041 & 5.610 \\
\hline 6 & 7 & 86.678 & 41.924 & 0.279 & 0.079 \\
\hline 6 & 8 & 910.287 & 435.669 & 25.403 & 10.929 \\
\hline 8 & 9 & 830.885 & 398.586 & 26.870 & 11.561 \\
\hline 9 & 10 & 669.014 & 321.642 & 30.993 & 13.334 \\
\hline 10 & 11 & 285.373 & 137.962 & 1.860 & 0.800 \\
\hline 10 & 12 & 318.628 & 153.869 & 2.318 & 0.997 \\
\hline 11 & 14 & 142.767 & 69.065 & 0.315 & 0.135 \\
\hline 11 & 13 & 54.343 & 26.251 & 0.343 & 0.097 \\
\hline 12 & 16 & 87.467 & 42.149 & 1.067 & 0.303 \\
\hline 12 & 15 & 142.444 & 68.878 & 1.567 & 0.674 \\
\hline 14 & 17 & 54.159 & 26.222 & 0.159 & 0.068 \\
\hline 14 & 18 & 54.275 & 26.231 & 0.275 & 0.078 \\
\hline 15 & 19 & 86.877 & 42.050 & 0.477 & 0.205 \\
\hline
\end{tabular}




\subsection{8 node practical system}

This feeder is analyzed to find the merit order of the feeding nodes for load demand factor of 1.0. The merit order for the given loading condition is presented in Table 4. The loss values shown are for single iteration of the load flow. The node 6 is the best location for reactive power compensation. The total loss at node 6 is $90.651 \mathrm{~kW}$ and the percentage loss is 2.38. The node 37 is the worst location for reactive power compensation. The total loss at node 37 is $5526.224 \mathrm{~kW}$ and the loss percentage is 59.80. Figure 3 presents the merit order with every node as feeding point.

Table 4 : Merit order of the feeding point for the 38-node system

\begin{tabular}{|c|c|c|c|c|c|c|c|}
\hline $\begin{array}{c}\text { Merit } \\
\text { order }\end{array}$ & $\begin{array}{c}\text { Node } \\
\text { No. }\end{array}$ & $\begin{array}{c}\text { Total Losses } \\
(\mathrm{kW})\end{array}$ & $\%$ Loss & $\begin{array}{c}\text { Merit } \\
\text { order }\end{array}$ & $\begin{array}{c}\text { Node } \\
\text { No. }\end{array}$ & $\begin{array}{c}\text { Total Losses } \\
(\mathrm{kW})\end{array}$ & $\%$ Loss \\
\hline 1 & 6 & 90.651 & 2.38 & 20 & 11 & 431.602 & 10.41 \\
\hline 2 & 26 & 101.788 & 2.67 & 21 & 35 & 436.298 & 10.51 \\
\hline 3 & 7 & 103.720 & 2.72 & 22 & 20 & 442.890 & 10.65 \\
\hline 4 & 5 & 108.842 & 2.85 & 23 & 31 & 469.866 & 11.23 \\
\hline 5 & 27 & 118.930 & 3.10 & 24 & 38 & 484.791 & 11.54 \\
\hline 6 & 4 & 119.879 & 3.13 & 25 & 12 & 488.700 & 11.63 \\
\hline 7 & 3 & 134.327 & 3.49 & 26 & 34 & 518.935 & 12.26 \\
\hline 8 & 8 & 160.317 & 4.14 & 27 & 21 & 525.836 & 12.40 \\
\hline 9 & 23 & 170.117 & 4.38 & 28 & 32 & 532.209 & 12.53 \\
\hline 10 & 2 & 189.277 & 4.85 & 29 & 33 & 618.423 & 14.27 \\
\hline 11 & 28 & 194.238 & 4.97 & 30 & 22 & 705.733 & 15.96 \\
\hline 12 & 1 & 202.826 & 5.18 & 31 & 13 & 791.726 & 17.57 \\
\hline 13 & 19 & 210.051 & 5.35 & 32 & 14 & 963.415 & 20.59 \\
\hline 14 & 24 & 255.209 & 6.43 & 33 & 36 & 1082.558 & 22.56 \\
\hline 15 & 29 & 263.403 & 6.62 & 34 & 15 & 1197.901 & 24.38 \\
\hline 16 & 9 & 269.023 & 6.75 & 35 & 17 & 1431.682 & 27.82 \\
\hline 17 & 30 & 315.161 & 7.82 & 36 & 16 & 1632.895 & 30.53 \\
\hline 18 & 25 & 382.165 & 9.33 & 37 & 18 & 1801.854 & 32.66 \\
\hline 19 & 10 & 403.691 & 9.80 & 38 & 37 & 5526.224 & 59.80 \\
\hline
\end{tabular}

A single iteration is performed, the CPU time taken for a bigger system will also be in the range of millisecond. Reactive power compensation is provided at the best and worst nodes. The percentage loss without compensation is $5.177 \%$. The candidate location for reactive power compensation is the node number 6 . The node 6 is the location where the feeder loss is found to be the lowest. This location is considered as the feeding source. About 250kvar compensation is placed at node 10. The load flow is performed for a single iteration.

The forward flows and losses are given in Table 5. The percentage loss with compensation at node 6 is $4.221 \%$. Reactive power compensation of about 250kvar is placed at the worst node 
Table 5 : Forward flows and losses with reactive power compensation at the candidate location (node 6) of the 38 node system

\begin{tabular}{|c|c|c|c|c|c|}
\hline \multirow{2}{*}{$\begin{array}{c}\text { Node } \\
\text { From }\end{array}$} & Node & \multicolumn{2}{|c|}{ Forward Flows } & \multicolumn{2}{c|}{ Losses } \\
\cline { 3 - 6 } & 2 & $\mathrm{~kW}$ & $\mathrm{kvar}$ & $\mathrm{kW}$ & $\mathrm{kvar}$ \\
\hline 1 & 2 & 3878.739 & 1412.719 & 9.803 & 4.997 \\
\hline 2 & 3 & 3407.810 & 1186.649 & 40.266 & 20.509 \\
\hline 2 & 19 & 361.139 & 161.081 & 0.161 & 0.154 \\
\hline 3 & 4 & 2337.969 & 668.926 & 13.919 & 7.089 \\
\hline 3 & 23 & 939.571 & 457.211 & 3.168 & 2.165 \\
\hline 4 & 5 & 2204.053 & 581.839 & 12.898 & 6.569 \\
\hline 5 & 6 & 2131.157 & 545.272 & 26.135 & 22.561 \\
\hline 6 & 7 & 1094.877 & 529.557 & 1.878 & 8.216 \\
\hline 6 & 26 & 950.126 & 973.140 & 2.547 & 1.297 \\
\hline 7 & 8 & 893.000 & 421.345 & 4.746 & 1.575 \\
\hline 7 & 35 & 0.000 & 0.000 & 0.000 & 0.000 \\
\hline 8 & 9 & 688.253 & 319.770 & 4.100 & 2.946 \\
\hline 8 & 34 & 0.000 & 0.000 & 0.000 & 0.000 \\
\hline 9 & 10 & 624.157 & 296.827 & 3.492 & 2.475 \\
\hline 10 & 11 & 560.659 & 274.348 & 0.543 & 0.182 \\
\hline 11 & 12 & 515.111 & 244.165 & 0.864 & 0.286 \\
\hline 12 & 13 & 454.254 & 208.881 & 2.614 & 2.057 \\
\hline 12 & 36 & 0.000 & 0.000 & 0.000 & 0.000 \\
\hline 13 & 14 & 391.642 & 171.826 & 0.715 & 0.941 \\
\hline 14 & 15 & 270.923 & 90.881 & 0.350 & 0.312 \\
\hline 15 & 16 & 210.574 & 80.571 & 0.277 & 0.202 \\
\hline 16 & 17 & 150.298 & 60.369 & 0.247 & 0.329 \\
\hline 17 & 18 & 90.054 & 40.043 & 0.052 & 0.041 \\
\hline 18 & 37 & 0.000 & 0.000 & 0.000 & 0.000 \\
\hline 19 & 20 & 270.976 & 120.925 & 0.832 & 0.749 \\
\hline 20 & 21 & 180.143 & 80.174 & 0.101 & 0.118 \\
\hline 21 & 22 & 90.046 & 40.060 & 0.044 & 0.058 \\
\hline 23 & 24 & 846.407 & 405.050 & 5.122 & 4.044 \\
\hline 24 & 25 & 421.280 & 201.002 & 1.282 & 1.003 \\
\hline 25 & 38 & 0.000 & 0.000 & 0.000 & 0.000 \\
\hline 26 & 27 & 887.597 & 946.851 & 3.260 & 1.660 \\
\hline 27 & 28 & 824.332 & 920.189 & 11.066 & 9.757 \\
\hline 28 & 29 & 753.269 & 890.434 & 7.671 & 6.685 \\
\hline 29 & 30 & 625.600 & 813.750 & 3.815 & 1.943 \\
\hline 30 & 31 & 421.784 & 211.807 & 1.560 & 1.542 \\
\hline 31 & 32 & 270.218 & 140.259 & 0.209 & 0.243 \\
\hline 32 & 33 & 60.014 & 40.022 & 0.013 & 0.020 \\
\hline
\end{tabular}


Table 6: Forward flows and losses with reactive power compensation at the worst location (node 37) of the 38 node system

\begin{tabular}{|c|c|c|c|c|c|}
\hline \multirow{2}{*}{$\begin{array}{l}\text { Node } \\
\text { From }\end{array}$} & \multirow{2}{*}{$\begin{array}{l}\text { Node } \\
\text { To }\end{array}$} & \multicolumn{2}{|c|}{ Forward Flows } & \multicolumn{2}{|c|}{ Losses } \\
\hline & & $\mathrm{kW}$ & kvar & $\mathrm{kW}$ & kvar \\
\hline 1 & 2 & 3921.055 & 1449.833 & 10.054 & 5.125 \\
\hline 2 & 3 & 3449.840 & 1223.618 & 41.435 & 21.104 \\
\hline 2 & 19 & 361.131 & 161.072 & 0.161 & 0.154 \\
\hline 3 & 4 & 2378.837 & 705.302 & 14.497 & 7.383 \\
\hline 3 & 23 & 939.583 & 457.220 & 3.170 & 2.166 \\
\hline 4 & 5 & 2244.328 & 617.913 & 13.460 & 6.855 \\
\hline 5 & 6 & 2170.873 & 581.059 & 27.304 & 23.570 \\
\hline 6 & 7 & 1133.371 & -435.702 & 1.876 & 8.204 \\
\hline 6 & 26 & 950.193 & 973.190 & 2.552 & 1.300 \\
\hline 7 & 8 & 931.491 & -543.907 & 5.614 & 1.863 \\
\hline 7 & 35 & 0.000 & 0.000 & 0.000 & 0.000 \\
\hline 8 & 9 & 725.879 & -645.771 & 6.642 & 4.772 \\
\hline 8 & 34 & 0.000 & 0.000 & 0.000 & 0.000 \\
\hline 9 & 10 & 659.237 & -670.543 & 6.329 & 4.486 \\
\hline 10 & 11 & 592.920 & -695.023 & 1.128 & 0.379 \\
\hline 11 & 12 & 546.777 & -725.407 & 2.126 & 0.703 \\
\hline 12 & 13 & 484.656 & -761.109 & 8.237 & 6.481 \\
\hline 12 & 36 & 0.000 & 0.000 & 0.000 & 0.000 \\
\hline 13 & 14 & 416.423 & -802.586 & 3.044 & 4.007 \\
\hline 14 & 15 & 293.379 & -886.593 & 3.526 & 3.139 \\
\hline 15 & 16 & 229.847 & -899.736 & 4.397 & 3.203 \\
\hline 16 & 17 & 165.451 & -922.939 & 7.689 & 10.266 \\
\hline 17 & 18 & 97.761 & -953.205 & 4.476 & 3.510 \\
\hline 18 & 37 & 3.288 & -996.712 & 3.287 & 3.287 \\
\hline 19 & 20 & 270.976 & 120.925 & 0.832 & 0.749 \\
\hline 20 & 21 & 180.149 & 80.181 & 0.101 & 0.118 \\
\hline 21 & 22 & 90.044 & 40.058 & 0.044 & 0.058 \\
\hline 23 & 24 & 846.406 & 405.049 & 5.124 & 4.046 \\
\hline 24 & 25 & 421.285 & 201.005 & 1.283 & 1.004 \\
\hline 25 & 38 & 0.000 & 0.000 & 0.000 & 0.000 \\
\hline 26 & 27 & 887.656 & 946.897 & 3.267 & 1.663 \\
\hline 27 & 28 & 824.381 & 920.229 & 11.088 & 9.776 \\
\hline 28 & 29 & 753.293 & 890.453 & 7.686 & 6.698 \\
\hline 29 & 30 & 625.610 & 813.757 & 3.822 & 1.947 \\
\hline 30 & 31 & 421.785 & 211.808 & 1.563 & 1.545 \\
\hline 31 & 32 & 270.227 & 140.269 & 0.209 & 0.244 \\
\hline 32 & 33 & 60.011 & 40.017 & 0.013 & 0.020 \\
\hline
\end{tabular}




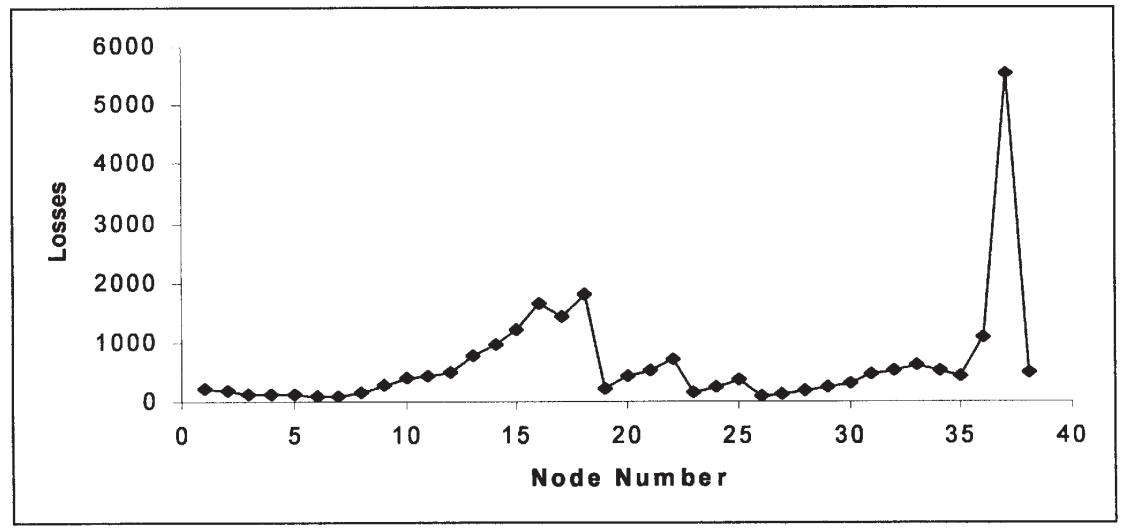

Figure 3 : Merit order of the feeding point for the 38 node system

37. The load flow is performed for a single iteration. The forward flows and losses are given in Table 6. The percentage loss with compensation at node 37 is $5.255 \%$.

\section{CONCLUSIONS}

The proposed technique is efficient and robust for analyzing practical and large distribution network having multi-conductor, multi-feeder with high $\mathrm{r} / \mathrm{x}$ ratio. The technique always guarantees convergence and it is suitable to real time operation of distribution network. A three-phase load flow solution technique based on backward and forward propagation and its applications to reactive power compensation and network reconfiguration has been demonstrated. In the case of feeder problems, to bring down the unbalance or fault occurrence, feeder reconfiguration and reactive power compensation can be done on-line using the proposed algorithm. Several practical large distribution feeders with multi-conductors and multi-feeders have been analyzed. The proposed method is computationally very fast, efficient and robust.

\section{REFERENCES}

1. Civanlar, S., Grainger, J.J., Yin, H. and Lee, S.S.H. (1988), IEEE Transaction on PWRD, vol. 3, no. 3, pp. 1217-1223.

2. Goswami, S.K and Basu, S.K.(1992), IEEE Transaction on Power Delivery, vol 7, no. 3, pp. 1484-1491.

3. Mesut, E.B., Felix F.W.(1989), IEEE Transaction on Power Delivery, vol. 4, no. 1, pp. 725-734.

4. Jin-Cheng, W., Hsiao-Dong, C., Karen, N.M. and Gary, D.(1997), IEEE Transaction on Power Delivery, vol 12, no. 2, pp. 953-958.

5. Thukaram, D., Jerome, J. and Wijekoon, B.H.K.(1999), Electrical Power Systems Research, vol 50, no. 3, pp. 227-236. 